TR ANSACTIONS OF THE

AMERICAN MATHEMATICAL SOCIETY

Volume 181 , July 1973

\title{
DEFORMING COHOMOLOGY CLASSES
}

\author{
BY
}

\author{
JOHN J. WAVRIK
}

\begin{abstract}
Let $\pi: X \rightarrow S$ be a flat proper morphism of analytic spaces. $\pi$ may be thought of as providing a family of compact analytic spaces, $X_{s}$, parametrized by the space $S$. Let $\mathcal{F}$ be a coherent sheaf on $X$ flat over $S$. $\mathcal{F}$ may be thought of as a family of coherent sheaves, $\mathcal{F}_{s}$, on the family of spaces $X_{s}$. Let $o \in S$ be a fixed point, $\xi_{o} \in H q\left(X_{o}, \mathcal{F}_{o}\right)$. In this paper, we consider the problem of extending $\xi_{0}$ to a cohomology class $\xi \epsilon$ $H q\left(\pi^{-1}(U), \mathfrak{F}\right)$ where $U$ is some neighborhood of $o$ in $S$. Extension problems of this type were first considered by P. A. Griffiths who obtained some results in the case in which the morphism $\pi$ is simple and the sheaf $\mathcal{F}$ is locally free. We obtain generalizations of these results without the restrictions. Among the applications of these results is a necessary and sufficient condition for the existence of a space of moduli for a compact manifold. This application was discussed in an earlier paper by the author. We use the Grauert "direct image" theorem, the theory of Stein compacta, and a generalization of a result of M. Artin on solutions of analytic equations to reduce the problem to an algebraic problem. In $\$ 2$ we discuss obstructions to deforming $\xi_{0}$; in $\$ 3$ we show that if no obstructions exist, $\xi_{0}$ may be extended; in $\$ 4$ we give a useful criterion for no obstructions; and in $\$ 5$ we discuss some examples.
\end{abstract}

Introduction. Let $\pi: X \rightarrow S$ be a flat proper morphism of analytic spaces, $o \in S$ a fixed point (so $X / S$ is a family of compact analytic spaces), $\mathcal{F}$ a coherent sheaf on $X$ flat over $S, \xi_{o} \in H^{q}\left(X_{0}, \mathcal{F}_{0}\right)$. In this paper, we study the problem of extending $\xi_{0}$ to a cohomology class $\xi \in H^{q}\left(\pi^{-1}(U), \mathcal{F}\right)$ where $U$ is a neighborhood of $o \in S$. This paper generalizes the results of Griffiths [4] in two ways: The morphism $\pi$ is not required to be simple (i.e. the family need not be a family of manifolds) and $\mathcal{F}$ need not be locally free. We obtain results similar to those of Griffiths in the more restricted case.

1. Reduction to an algebraic problem. We use the Grauert "direct image" theorem [3] and the theory of Stein compacta (Riemenschneider [7], also Frisch [1]) to reduce the deformation problem to an algebraic problem.

Let $\pi: X \rightarrow S$ be a flat proper morphism of analytic spaces, o $\in S, \mathcal{F}$ coherent on $X$ flat over $S$. It is well known [2, Théorème 4.11.1] that $\underset{\longrightarrow}{\lim } H^{q}\left(\pi^{-1}(U), \mathcal{F}\right)=H^{q}\left(X_{o}, \mathcal{F}\right)$. So extending $\xi_{o} \in H^{q}\left(X_{o}, \mathcal{F}_{o}\right)$ to some neighbor-

Received by the editors August 15, 1972.

AMS (MOS) subject classifications (1970). Primary 32G05; Secondary 32C 35.

Key words and phrases. Extension problem, deformation theory, families of analytic spaces, obstructions, space of moduli. 
hood of $o$ is equivalent to extending to $H^{q}\left(X_{o}, \mathcal{F}\right)$.

We recall the following definition:

Definition 1.1 [7]. A subset $K$ of a complex space $X$ is called a Stein compactum if

(i) $K$ is compact,

(ii) $K$ is semianalytic,

(iii) $K$ has a fundamental system of open neighborhoods in $X$ which are Stein spaces.

The main properties of Stein compacta which we need are the following:

Proposition 1.2. Let $X$ be an analytic space, $K \subset X$ a Stein compactum, $\mathcal{F}$ a coberent sheaf on $X$

(i) $\Gamma(K, \mathcal{O})$ is a noetherian C-algebra,

(ii) $\Gamma(K, \mathcal{F})$ is a finite $\Gamma(K, \mathcal{O})$-module,

(iii) $H^{q}(K, \mathcal{F})=0$ if $q \geq 1$.

Proof. See [7].

Now let $\left\{K_{i}^{\prime}\right\}$ be a finite cover of $X_{o}$ by Stein compacta in $X$. Let $K_{i}=$ $X_{o} \cap K_{i}^{\prime}$. Then $\mathcal{K}=\left\{K_{i}\right\}$ is a finite cover of $X_{\mathrm{o}}$ by Stein compacta. Leray's theorem [2, Corollary to Theorem 5.2.4] shows that $H^{q}\left(X_{o}, \mathcal{F}\right)=H^{q}(\mathcal{K}, \mathcal{F})$. Thus we have a complex

$$
0 \rightarrow C^{0}(\mathcal{K}, \mathcal{F}) \rightarrow \cdots \rightarrow C^{m}(\mathcal{K}, \mathcal{F}) \rightarrow 0
$$

of flat $A$-modules $\left(A=\mathcal{O}_{S, o}\right)$ and $H^{q}\left(X_{o}, \mathcal{F}\right)=H^{q}(C \cdot(\mathcal{K}, \mathcal{F}))$.

Let $T$ be an analytic space, $o \in T$, and $g:(T, o) \rightarrow(S, o)$ a finite morphism. Let $X^{\prime}=X \times{ }_{S} T, \pi^{\prime}: X^{\prime} \rightarrow T$, and $\mathcal{F}^{\prime}$ the pullback of $\mathcal{F}$ to $X^{\prime}$. Then $X^{\prime} / T$ is flat and proper and represents the family induced by $g . \mathcal{F}^{\prime}$ is the induced sheaf. We may identify $X_{o}^{\prime}=\pi^{\prime-1}(o)$ with $X_{o} \cdot K_{i}$ is still a Stein compactum in $X^{\prime}[7,(2.20)]$. Let $B=\mathcal{Q}_{T, o^{\circ}} B$ is a finite $A$-algebra. We have $\Gamma\left(K_{i}, \mathcal{F}^{\prime}\right)=$ $\Gamma\left(K_{i}, \mathcal{F}\right) \otimes_{A} B[7,(2.21)]$ and so $C^{q}\left(\mathcal{K}, \mathcal{F}^{\prime}\right)=C^{q}(\mathcal{K}, \mathcal{F}) \otimes_{A} B$.

Proposition 1.3. $H^{q}(C \cdot(\mathcal{K}, \mathcal{F}))$ is a finite A-module.

Proof. We have already observed that

$$
H^{q}\left(C^{\cdot}(\mathcal{K}, \mathcal{F})\right)=H^{q}(X, \mathcal{F})=\underline{\lim }_{o} H^{q}\left(\pi^{-1}(U), \mathcal{F}\right)=\left(R^{q} \pi_{*}(\mathcal{F})\right)_{0} .
$$

By the direct image theorem [3], $R^{q} \pi_{*}(\mathcal{F})$ is a coherent sheaf on $X$, thus $\left(R^{q} \pi_{*}(\mathcal{F})\right)_{0}$ is a finite $A$-module.

Now we show that the complex $C^{\circ}=C^{\bullet}(\mathcal{K}, \mathfrak{F})$ can be replaced by a complex of finite free $A$-modules. 
Proposition 1.4. Let $A$ be a noetherian ring, $C^{\cdot}$ a complex of A-modules, $C^{q}=0$ if $q>m, H^{q}\left(C^{*}\right)$ a finite $A$-module for all $q$. Then there is a complex $D^{\cdot}$ of finite free A-modules, $D^{q}=0$ if $q>m$, and a morphism $\phi^{*}: D^{\cdot} \rightarrow C^{\cdot}$ such that the induced morphisms $H^{q}\left(D^{\cdot}\right) \rightarrow H^{q}\left(C^{\cdot}\right)$ are isomorphisms.

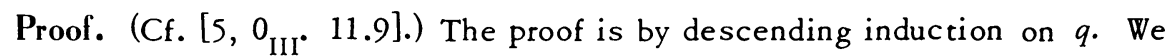
begin by choosing $D^{m}$ a finite free $A$-module together with an epimorphism $\psi^{m}$ : $D^{m} \rightarrow H^{m}\left(C^{\cdot}\right)$. $\psi^{m}$ can be lifted to $\phi^{m}: D^{m} \rightarrow C^{m}$. Now suppose that we have a commutative diagram:

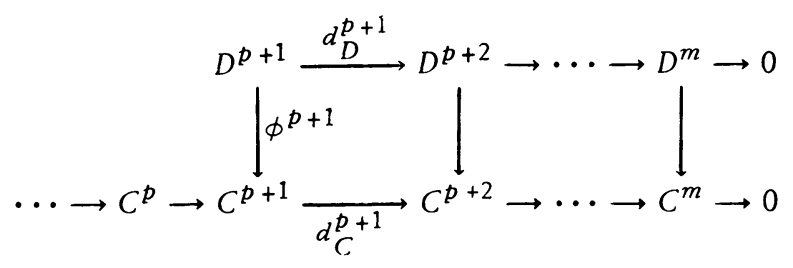

with the $D^{i}$ finite free $A$-modules such that

(i) $\phi^{j}$ induces an isomorphism $H^{j}\left(D^{\cdot}\right) \rightarrow H^{j}\left(C^{\cdot}\right), j \geq p+2$,

(ii) $\phi^{p+1}$ induces an epimorphism $\operatorname{Ker}\left(d_{D}^{p+1}\right) \rightarrow H^{p+1}\left(C^{\cdot}\right)$.

We have produced this situation for $p=m-1$ above.

Let $Z^{q}=\operatorname{Ker} d_{C}^{q}, B^{q}=\operatorname{Im} d_{C}^{q}, Z^{\prime q}=\operatorname{Ker} d_{D}^{q} . \phi^{p+1}$ induces an epimorphism $Z^{\prime p+1} \rightarrow H^{p+1}\left(C^{\cdot}\right)$, let $K^{p+1}$ denote the kernel. We have

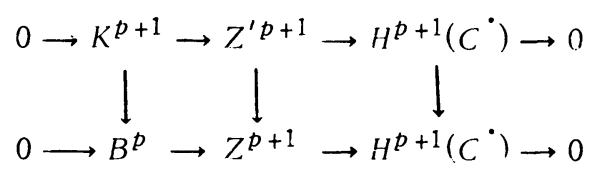

commutative with exact rows. Since $A$ is noetherian, $Z^{\prime p+1}$ and $K^{p+1}$ are finite $A$-modules. Thus we may find a finite free $A$-module $F^{\prime \prime}$ and an epimorphism $F^{\prime \prime} \rightarrow K^{p+1}$. We lift the composition $F^{\prime \prime} \rightarrow K^{p+1} \rightarrow B^{p}$ to a morphism $F^{\prime \prime} \rightarrow C^{p}$. Let $F^{\prime}$ be a finite free $A$-module with an epimorphism $F^{\prime} \rightarrow Z^{p / B^{p-1}}=H^{p}\left(C^{\cdot}\right)$. We lift this latter morphism to a morphism $F^{\prime} \rightarrow Z^{p}$, hence, by composition, we obtain $F^{\prime} \rightarrow C^{p}$. Let $D^{p}=F^{\prime} \times F^{\prime \prime}, \phi^{p}$ the map induced by $F^{\prime} \rightarrow C^{p}$, $F^{\prime \prime} \rightarrow C^{p}$ and $d_{D}^{p}$ the composition $F^{\prime} \times F^{\prime \prime} \rightarrow F^{\prime \prime} \rightarrow K^{p+1} \rightarrow D^{p+1}$. We may verify that with these choices conditions (i) and (ii) are satisfied with $p$ replaced by $p-1$. By induction we obtain $D^{*}, \phi^{\circ}: D^{*} \rightarrow C^{\cdot}$ as required.

Proposition 1.5. Let $A$ be a noetherian ring, $D ; C$ complexes of flat $A$ modules with $D^{q}, C^{q}=0, q>m, \phi^{\circ}: D^{\cdot} \rightarrow C^{\cdot}$ a morphism of complexes inducing isomorphisms $H^{q}\left(D^{\cdot}\right) \rightarrow H^{q}\left(C^{\cdot}\right)$. Then for every $A$-module $M, \phi^{\cdot} \otimes 1$ induces isomorphisms $H^{q}\left(D^{\cdot} \otimes_{A} M\right) \rightarrow H^{q}\left(C \cdot \otimes_{A} M\right)$. 
Proof. We define a complex $L^{\cdot}$ by

$$
L^{q}=C^{q-1} \oplus D^{q}, \quad d_{L}=\left(\begin{array}{cc}
-d_{C} & \phi \\
0 & d_{D}
\end{array}\right) .
$$

We easily check that $d_{L} \circ d_{L}=0$. If $C^{q}[-1]=C^{q-1}, C^{\cdot}[-1]$ is a complex with $H^{q}\left(C^{\cdot}[-1]\right)=H^{q-1}\left(C^{\cdot}\right)$. We have an exact sequence of complexes $0 \rightarrow C^{\cdot}[-1]$

$\rightarrow L^{\cdot} \rightarrow D^{\bullet} \rightarrow 0$ and so an exact cohomology sequence

$$
\cdots \rightarrow H^{q}\left(C^{\cdot}[-1]\right) \rightarrow H^{q}\left(L^{*}\right) \rightarrow H^{q}\left(D^{\circ}\right) \stackrel{\delta}{\longrightarrow} H^{q+1}\left(C^{\cdot}[-1]\right) \rightarrow \cdots
$$

If we identify $H^{q+1}\left(C^{\cdot}[-1]\right)$ with $H^{q}\left(C^{\cdot}\right)$, the map $\delta$ is identified with the map induced by $\phi^{\circ}$. Thus $\phi^{*}$ induces isomorphisms iff $H^{q}\left(L^{\circ}\right)=0$ for all $q$. Let $Z^{q}=\operatorname{Ker} d_{L}^{q}, H^{q}\left(L^{\cdot}\right)=0$ iff $0 \rightarrow Z^{q-1} \rightarrow L^{q-1} \rightarrow Z^{q} \rightarrow 0$ is exact. The complex constructed in this way for $\phi^{\circ} \otimes_{A} 1$ is $L^{\cdot} \otimes_{A} M$. Thus the induced morphisms are isomorphisms iff $0 \rightarrow Z^{q-1} \otimes_{A} M \rightarrow L^{q-1} \otimes_{A} M \rightarrow Z^{q} \otimes_{A} M \rightarrow 0$ is exact for all $q$. Now $L^{q}$ is flat for all $q$. Since $L^{m+2}=0, Z^{m+1}=L^{m+1}$ is flat. By descending induction, $Z^{q}$ is flat for all $q$.

By hypothesis, $0 \rightarrow Z^{q-1} \rightarrow L^{q-1} \rightarrow Z^{q} \rightarrow 0$ is exact for all $q$. By flatness, the same is true for the sequence $0 \rightarrow Z^{q-1} \otimes_{A} M \rightarrow L^{q-1} \otimes_{A} M \rightarrow Z^{q} \otimes_{A} M \rightarrow$ 0 . The result follows.

To summarize, we have

Theorem 1.6. Let $\pi: X \rightarrow S$ be a flat proper morphism, o $\in S, \mathcal{F}$ a coberent sheaf on $X$ flat over $S, A=\mathcal{O}_{S, o^{\circ}}$. Then there is a complex $D^{\circ}$ of finite free $A$ modules, $D^{q}=0$ for $q>m$, such that for each finite morphism $g:(T, o) \rightarrow(S, o)$ we have a natural isomorphism

$$
H^{q}\left(D^{\cdot} \otimes_{A} B\right) \rightarrow \underline{\lim } H^{q}\left(\pi^{\prime-1}(U), \mathcal{F}^{\prime}\right),
$$

where $\mathcal{F}^{\prime}$ is the pullback of $\mathcal{F}$ to $X^{\prime}=X \times_{S} T, B=\mathcal{O}_{T, 0}$, and $U$ runs over neigbborboods of $o \in T$.

In particular, $H^{q}\left(D^{\cdot} \otimes_{A} \mathrm{C}\right)$ is naturally isomorphic to $H^{q}\left(X_{o^{\prime}}, \mathcal{F}_{o}\right)$.

2. Obstructions to deforming a cohomology class. In this section we will study the problem of extending $\xi_{0} \in H^{q}\left(X_{o}, \mathcal{F}_{o}\right)$ to infinitesimal neighborhoods of $o \in S$.

Recall that if $S$ is an analytic space, $o \in S$, the (nonreduced) analytic space $S^{(n)}=\left(\{o\}, \mathcal{O}_{S, o} / \mathrm{m}^{n+1}\right)$ is called the $n$th infinitesimal neighborhood of $o$. We let $A^{(n)}=A / m^{n+1}, X^{(n)}=X \times S_{S} S^{(n)}, \mathcal{F}(n)$ the pullback of $\mathcal{F}$ to $X^{(n)}$.

Using the analysis of the previous section, $H^{q}\left(X^{(n)}, \mathcal{F}(n)\right)$ is naturally isomorphic to $H^{q}\left(D^{\cdot} \otimes_{A} A^{(n)}\right)$. We have $\xi_{0} \in H^{q}\left(D^{\cdot} \otimes_{A} A^{(0)}\right)$. We now seek to extend 
$\xi_{o}$ sucessively to $\xi_{n} \in H^{q}\left(D^{\cdot} \otimes_{A} A^{(n)}\right)$. Suppose, in fact, that $\xi_{n-1} \epsilon$ $H^{q}\left(D \cdot \otimes_{A} A^{(n-1)}\right)$. We have an exact sequence of $A$-modules

$$
0 \rightarrow \mathrm{m}^{n} / \mathrm{m}^{n+1} \rightarrow A^{(n)} \rightarrow A^{(n-1)} \rightarrow 0
$$

and so obtain an exact sequence of complexes

$$
0 \rightarrow D^{\cdot} \otimes_{A} \mathrm{~m}^{n} \cdot \mathrm{m}^{n+1} \rightarrow D^{\cdot} \otimes_{A} A^{(n)} \rightarrow D^{\cdot} \otimes_{A} A^{(n-1)} \rightarrow 0
$$

and therefore an exact cohomology sequence

$$
\cdots \rightarrow H^{q}\left(D^{\cdot} \otimes_{A} A^{(n)}\right) \rightarrow H^{q}\left(D^{\cdot} \otimes_{A} A^{(n-1)}\right) \rightarrow H^{q+1}\left(D^{\cdot} \otimes_{A} \mathrm{~m}^{n} / \mathrm{m}^{n+1}\right) \rightarrow \cdots
$$

Thus $\xi_{n-1} \in H^{q}\left(D^{\cdot} \otimes_{A} A^{(n-1)}\right)$ may be lifted to $H^{q}\left(D^{\cdot} \otimes_{A} A^{(n)}\right)$ if and only if its image $\omega\left(\xi_{n-1}\right) \in H^{q+1}\left(D \cdot \otimes_{A} \mathrm{~m}^{n} / \mathrm{m}^{n+1}\right)$ vanishes. Thus $\omega\left(\xi_{n-1}\right)$ represents the obstruction to extending $\xi_{n-1}$.

Now $H^{q+1}\left(D \cdot \otimes_{A} \mathrm{~m}^{n} / \mathrm{m}^{n+1}\right)$ is naturally isomorphic to $H^{q+1}\left(X_{o}, \mathcal{F}_{0} \otimes_{C} \mathrm{~m}^{n} / \mathrm{m}^{n+1}\right)$ which is a direct sum of copies of $H^{q+1}\left(X_{o}, \mathcal{F}_{o}\right)$. We have proved

Proposition 2.1. The nth obstruction to extending $\xi_{0} \in H^{q}\left(X_{0}, \mathcal{F}_{0}\right)$ lies in $H^{q+1}\left(X_{o}, \mathcal{F}_{0} \otimes \mathrm{m}^{n} / \mathrm{m}^{n+1}\right)$.

Corollary. If $H^{q+1}\left(X_{o}, \mathcal{F}_{o}\right)=0$, there are no obstructions to extending $\xi_{0} \epsilon$ $H^{q}\left(X_{o}, \mathcal{F}_{o}\right)$.

We will now show that for a given $X / S, \mathcal{F}$ and $q$, only finitely many obstructions exist to extending any $\xi_{0} \in H^{q}\left(X_{o}, \mathcal{F}_{o}\right)$.

Proposition 2.2. With $X, S, \mathcal{F}, q$ as above, there is a positive integer $n_{0}$ such that if $\xi_{0} \in H^{q}\left(X_{0}, \mathcal{F}_{0}\right)$ can be extended to $H^{q}\left(X^{\left(n_{0}\right)}, \mathcal{F}^{\left(n_{0}\right)}\right)$ then it can be extended to $H^{q}\left(X^{(n)}, \mathscr{F}^{(n)}\right)$ for all $n$.

Proof. $H^{q}\left(D \cdot \otimes_{A} A^{(n)}\right)=M_{n}$ is a finite $A^{(n)}$-module, hence a finite dimensional vector space over C. If $n \geq m$, the morphism $M_{n} \rightarrow M_{m}$ is $A$-linear, hence C-linear. Let $M_{n}^{\prime}=\operatorname{Im}\left(M_{n} \rightarrow M_{0}\right) . M_{n}^{\prime}$ is a subspace of $M_{0}$ and we have $M_{n}^{\prime}$ ว $M_{n+1}^{\prime}$. Since $M_{0}{ }^{n}$ is finite dimensional, there is an $n_{0}$ such that $M_{n}^{\prime}=M_{n}^{\prime}$ for $n \geq n_{o}$. This means, however, that any $\xi_{0} \in M_{0}$ which can be extended to $M_{n_{0}}$ can be extended to $M_{n}$ for all $n$.

3. Formal deformations give actual deformations. If $\xi_{0} \in H^{q}\left(X_{o}, \mathcal{F}_{o}\right)$ has no obstructions, it can be extended to $\xi_{n} \in H^{q}\left(X^{(n)}, \mathcal{F}(n)\right)$ for all $n$. This stepby-step extension is analogous to determining a power series term-by-term. In the power series case there is no guarantee of convergence. In this section we will show that if $\xi_{o}$ has no obstructions (i.e. can be formally extended) then it 
also can be actually extended to a $\xi \in H^{q}\left(\pi^{-1}(U), \mathcal{F}\right)$ for some neighborhood $U$ $\ni$ o.

Let $A$ be an analytic C-algebra, $\phi: A^{m} \rightarrow A^{n}$. If $B$ is an analytic $A$-algebra, $\phi$ induces $\phi_{B}: B^{m} \rightarrow B^{n}$. If $\sigma: A \rightarrow B$ is the structure morphism, and $\phi$ is given by a matrix $\left(\alpha_{j}^{i}\right)$ then $\phi_{B}$ is given by the matrix $\left(\sigma\left(\alpha_{j}^{i}\right)\right)$. If $\lambda: B \rightarrow C$ is a morphism, $\lambda$ induces $\lambda: \operatorname{Ker} \phi_{B} \rightarrow \operatorname{Ker} \phi_{C}$. If $k \in \operatorname{Ker} \phi_{B}$ we denote by $\bar{k}$ its image in $\operatorname{Ker} \phi_{C}$.

Let $a \in \operatorname{Ker} \phi_{C}$ and set $K(B)=\left\{k \in \operatorname{Ker} \phi_{B} \mid \vec{k}=a\right\} . K$ is a covariant functor from the category of analytic $A$-algebras to the category of sets.

Proposition 3.1. $K$ is representable.

Proof. Let $a=\left(a_{1}, \ldots, a_{m}\right) \in \operatorname{Ker} \phi_{\mathbf{C}} \subset \mathbf{C}^{m}$. Let $\phi$ be given by the matrix $\left(\alpha_{j}^{i}\right)$. Since $A$ is an analytic $\mathbf{C}$-algebra, we have $A=\mathbf{C}\left\{x_{1}, \ldots, x_{p}\right\} /\left(f_{1}, \ldots, f_{a}\right)$. Lift $\alpha_{j}^{i}$ to $\mathbf{C}\{x\}$. Let $R=\mathbf{C}\left\{x ; z_{1}, \ldots, z_{m}\right\} /\left(f_{i} ; \Sigma \alpha_{j}^{i}\left(z_{i}+a_{i}\right)\right) . R$ is an analytic $A$-algebra. We have $\phi_{R}(\xi)=\Sigma a_{j}^{i}\left(z_{i}+a_{i}\right)=0$ where $\xi=\left(\xi_{1}, \ldots, \xi_{m}\right) \in R^{m}$, $\xi_{i}=z_{i}+a_{i}$. Thus $\xi \in \operatorname{Ker} \phi_{R}$. Moreover, $\bar{\xi}=a$, so $\xi \in K(R)$. We will now show that $(R, \xi)$ represents $K$. Let $B$ be any analytic $A$-algebra, $k \in K(B), k=$ $\left(k_{1}, \ldots, k_{m}\right) \in B^{m} \cdot k_{i}-a_{i} \in \mathrm{m}_{B}$ so there is a unique morphism of $A$-algebras $A\left\{z_{1}, \ldots, z_{m}\right\} \rightarrow B$ which carries $z_{i}$ to $k_{i}-a_{i}$ (here $A\left\{z_{1}, \cdots, z_{m}\right\}=$ $\left.\mathrm{C}\left\{x ; z_{1}, \cdots, z_{m}\right\} /\left(f_{i}\right)\right)$. Since $k \in \operatorname{Ker} \phi_{B}$ this induces a unique morphism $f: R$ $\rightarrow B$ such that $K(f)(\xi)=k$.

Let $D^{\cdot}$ be a complex of finite free $A$-modules, let $\xi_{o} \in H^{q}\left(D \cdot \otimes_{A} \mathrm{C}\right)$. We set

$$
F(B)=\operatorname{Ker}\left(D^{q} \otimes_{A} B \rightarrow D^{q+1} \otimes_{A} B\right), \quad G(B)=H^{q}\left(D^{\cdot} \otimes_{A} B\right) .
$$

$F, G$ are covariant functors on the category of analytic $A$-algebras; $F(B), G(B)$ are $B$-modules, and we have a natural transformation $u: F \rightarrow G$ such that $u(B)$ is a surjective $B$-module homomorphism for each $B$. Let $G_{\xi_{o}}(B)=\{\lambda \in G(B) \mid$ $\left.G\left(p_{B}\right)(\lambda)=\xi_{o}\right\}$ where $p_{B}: B \rightarrow \mathrm{C}$ is the natural map. If $\eta_{o} \in u(C)-1\left(\xi_{o}\right)$ we set $F_{\eta_{0}}(B)=\left\{\sigma \in F(B) \mid F\left(p_{B}\right)(\sigma)=\eta_{o}\right\}, F_{\eta_{0}}$ and $G_{\xi_{0}}$ are also covariant functors on the category of analytic $A$-algebras. $u$ induces $u: F_{\eta_{0}} \rightarrow G_{\xi_{o}}$.

From Proposition 3.1 it follows that $F_{\eta_{0}}$ is representable for any $\eta_{0}$.

Proposition 3.2. If $G_{\xi_{o}}\left(A^{(n)}\right) \neq \varnothing$ for all $n$, then $G_{\xi_{o}}(A) \neq \varnothing$.

Proof. $F(\mathbf{C})$ is a finite dimensional vector space over $\mathbf{C}$ and $u(\mathbf{C}): F(\mathbf{C}) \rightarrow$ $G(\mathbf{C})$ is $\mathbf{C}$-linear, so $S=u^{-1}(\mathbf{C})\left(\xi_{o}\right)$ is an affine variety in $F(\mathbf{C}) . F\left(p_{A}(n)\right.$ : $F\left(A^{(n)}\right) \rightarrow F(\mathbf{C})$ is $A$-linear hence $\mathbf{C}$-linear, so $I_{n}=\operatorname{Im}\left(F\left(p_{A}(n)\right)\right)$ is a subspace of $F(\mathrm{C})$. Let $S_{n}=I_{n} \cap S$. $S_{n}$ is an affine variety and $S_{n} \supset S_{n+1}$. By hypothesis, $G_{\xi_{o}}\left(A^{(n)}\right) \neq \varnothing$, hence $S_{n} \neq \varnothing$. From [9, Lemma 2.1] it follows that $\bigcap S_{n} \neq \varnothing$. 
Thus we can find $\eta_{0} \in u(C)^{-1}\left(\xi_{o}\right)$ such that $F_{\eta_{0}}\left(A^{(n)}\right) \neq \varnothing$ for all $n$. We have

Lemma 3.3. Let $F$ be a covariant functor from the category of analytic $A$ algebras to the category of sets. Let $(R, \xi)$ be sucb that $\operatorname{Hom}_{A}(R, B) \rightarrow F(B)$ is surjective for all $B$. Then $F\left(B^{(n)}\right) \neq \varnothing$ for all $n$ implies $F(B) \neq \varnothing$.

Proof. If $F\left(B^{(n)}\right) \neq \varnothing$ then $\operatorname{Hom}_{A}\left(R, B^{(n)}\right) \neq \varnothing$. Let $f^{(n)} \in \operatorname{Hom}_{A}\left(R, B^{(n)}\right)$. By a generalization of a result of M. Artin which we proved in [9, Theorem 2.4], we can find an $f \in \operatorname{Hom}_{A}(R, B)$. Thus $\operatorname{Hom}_{A}(R, B) \neq \varnothing$ so $F(B) \neq \varnothing$.

Now with $\eta_{0}$ as chosen above, $F_{\eta_{0}}\left(A^{(n)}\right) \neq \varnothing$ for all $n$. But $F_{\eta_{0}}$ is representable. Hence, by the lemma, $F_{\eta_{0}}(A) \neq \varnothing$. But then also $G_{\xi_{o}}(A) \neq \varnothing$, which is what we wanted to prove.

Question. Is $G \xi_{o}$ representable? I.e., is there a universal family of extensions of $\xi_{0}$ ? This appears to be the case in many examples. If true, the proof of Proposition 3.2 can be simplified.

4. A useful criterion for no obstructions. We have a restriction map $H^{q}\left(\pi^{-1}(U), \mathcal{F}\right) \rightarrow H^{q}\left(X_{o}, \mathcal{F}_{o}\right)$ for any open neighborhood $U$ of $o$ in $S$. This provides us with a restriction map $R^{q} \pi_{*}(\mathcal{F})_{0} \rightarrow H^{q}\left(X_{o}, \mathcal{F}_{0}\right) \cdot m \cdot R^{q} \pi_{*}(\mathcal{F})_{0}$ is in the kernel, so we obtain

$$
t_{0}^{q}: R^{q} \pi_{*}(\mathcal{F})_{o} / \mathrm{m} \cdot R^{q} \pi_{*}(\mathfrak{F})_{0} \rightarrow H^{q}\left(X_{o}, \mathcal{F}_{o}\right) .
$$

If $t_{0}^{q}$ is surjective, then any class in $H^{q}\left(X_{0}, \mathcal{F}_{0}\right)$ is unobstructed. If $Q_{S, o}$ is reduced, we obtain a useful criterion for $t_{o}^{q}$ to be bijective. This criterion first appears in the paper of Grauert [3], a complete proof is given by Riemenschneider in $[7]$.

Theorem 4.1. Let $\pi: X \rightarrow S$ be a flat proper morphism of analytic spaces, $\mathcal{F}$ a coberent sheaf on $X$ flat over $S, o \in S$ a fixed point, and let $\mathcal{S}_{S, o}$ be reduced. If $\operatorname{dim}_{C} H^{q}\left(X_{s}, \mathcal{F}_{s}\right)$ is independent of $s$ in some neighborbood of o then $t_{o}^{q}$ and $t_{0}^{q-1}$ are bijective.

Proof. See [7, (3.5)].

Corollary 1. If $\operatorname{dim}_{\mathrm{C}} H^{q}\left(X_{s}, \mathcal{F}_{s}\right)$ is independent of $s$ in a neighborbood of $o$, any $\xi_{o} \in H^{q}\left(X_{o}, \mathcal{F}_{o}\right)$ is unobstructed.

Corollary 2. If $\operatorname{dim}_{\mathrm{c}} H^{q+1}\left(X_{s}, \mathcal{F}_{s}\right)$ is independent of $s$ in a neighborhood of $o$, any $\xi_{0} \in H^{q}\left(X_{0}, \mathscr{F}_{o}^{C}\right)$ is unobstructed.

Remarks. 1. " $\xi_{0}$ is unobstructed" means that $\xi_{o}$ may be extended to $\xi_{n}$ $H^{q}\left(X^{(n)}, \mathfrak{F}^{(n)}\right)$ for all $n$ or, equivalently, to $\xi \in H^{q}\left(\pi^{-1}(U), \mathfrak{F}\right)$ for some neighborhood $U$ of $o$. 
2. The proof of Theorem 4.1 is independent of the "formal-to-actual" machinery of $\$ 3$.

The converses of the two corollaries do not hold as examples in the next section show. We will prove the converse to Theorem 4.1 which is useful in identifying groups containing obstructed classes.

In Theorem 1.6 we constructed a complex $D^{\circ}$ of finite free $A=\mathcal{O}_{S, o^{-m o d u l e s . ~}}$ Using the notation of Grothendieck [5, III. 7.4.1] (with modifications, since we are considering a cohomology rather than homology functor) we introduce the cohomology functor $T^{\cdot}$ on the category of $A$-modules by $T^{q}(M)=H^{q}\left(D^{*} \otimes_{A} M\right)$. $T^{q}(M)$ is an $A$-module and we have an $A$-module homomorphism $t_{M}^{q}: T^{q}(A) \otimes_{A} M$ $\rightarrow T^{q}(M)$. If $M=\mathrm{C}, t_{M}^{q}$ coincides with $t_{o}^{q}$ defined above. We have

Proposition 4.2 ([5, III. 7.3.1]). The following are equivalent:

(a) $T^{q}$ is right exact.

(b) $T^{q+1}$ is left exact.

(c) $t_{M}^{q}$ is an isomorpbism for all $M$.

(d) $t_{M}^{q}$ is an epimorphism for all $M$.

(e) The canonical map $T^{q}(A) \rightarrow T^{q}(\mathbf{C})$ is an epimorphism (i.e. every class in $H^{q}\left(X_{0}, \mathcal{F}_{0}\right)$ is unobstructed $)$.

Further, we have

Proposition 4.3. The following are equivalent:

(a) $T^{q}$ is exact.

(b) $T^{q}$ is right exact and $T^{q}(A)$ is free.

(c) $T^{q-1}(A) \rightarrow T^{q-1}(\mathbf{C}), T^{q}(A) \rightarrow T^{q}(\mathbf{C})$ are surjective.

Proof. From [5, III. 7.5.4 and III. 7.5.7].

Theorem 4.4 (Converse to Theorem 4.1). Under the general conditions of Theorem 4.1, if $t_{o}^{q}$ and $t_{o}^{q-1}$ are bijective (or even surjective) then $\operatorname{dim}_{C} H^{q}\left(X_{s}, \mathcal{F}_{s}\right)$ is independent of $s$ in a neigbborbood of $o$.

Proof. Under the hypotheses, Proposition 4.3 shows that $T^{q}(A)=R^{q} \pi_{*}(\mathfrak{F})$ 。 is a free $A$-module. Since $R^{q} \pi_{*}(\mathcal{F})$ is coherent, it is free in some neighborhood of $o$. Thus $\operatorname{dim}_{C}\left(R^{q} \pi_{*}(\mathcal{F})_{s} / m_{s} \cdot R^{q} \pi_{*}(\mathcal{F})_{s}\right)$ is independent of $s$ in a neighborhood of o. Moreover, by Proposition 4.2, $t_{0}^{q}$ is bijective. From [3, $\S 7$, Theorem 2], $t_{s}^{q}$ is bijective for $s$ in a neighborhood of $o$. (If $S=\mathrm{C}$, this result may be proved by using the result of Frisch [1, Theorem IV, 9] together with [5, III. 7.4.3].) Thus $\operatorname{dim}_{\mathrm{C}} H^{q}\left(X_{s}, \mathcal{F}_{s}\right)$ is independent of $s$ in a neighborhood of $o$.

Corollary. $\operatorname{dim}_{\mathrm{C}} H^{0}\left(X_{s}, \mathcal{F}_{s}\right)$ is independent of $s$ in a neigbborbood of $o$ if and only if every class in $H^{0}\left(X_{o}, \mathcal{F}_{o}\right)$ is unobstructed. 
The criterion of Corollary 2 of Theorem 4.1 is essentially that obtained by Griffiths [4] in the more restricted case considered by him. We have shown in [8] that an extension problem of the type considered here is intimately related to the problem of whether a compact manifold has a space of moduli. In [8] we used the criteria of Corollaries 1 and 2 of Theorem 4.1. The corollary to Theorem $4.4 \mathrm{com}$ pletes the proof of a statement made in Remark 2 on p. 413 of [8] and so shows the necessity of the condition given there for the existence of a space of moduli.

It should be observed that the results of this section deal only with the situation in which all cohomology classes are unobstructed and the parameter space is reduced. In general, $H^{q}\left(X_{o}, \mathcal{F}_{o}\right)$ may contain both obstructed and unobstructed classes. Examples will be given in the next section.

5. Examples. Thase examples are based on a family of Hirzebruch surfaces discussed by Kodaira in [6]. Each $X_{s}$ is a $\mathbf{P}^{1}$-bundle over $\mathbf{P}^{1}, S=\mathbf{C}$. The family is obtained by patching together two copies of $\mathbf{C} \times \mathbf{P}^{1} \times \mathbf{C}$. We use local coordinates $\left(z_{i}, \zeta_{i}, s\right)$ in the $i$ th copy $(i=1,2)$. Here $\zeta_{i}$ is an inhomogeneous coordinate in $\mathbf{P}^{1}$. We identify $\left(z_{1}, \zeta_{1}, s\right)$ with $\left(z_{2}, \zeta_{2}, s\right)$ if and only if

$$
z_{1}=1 / z_{2}, \quad \zeta_{1}=z_{2}^{2} \zeta_{2}+s z_{2}
$$

$X_{s} \cong \mathbf{P}^{1} \times \mathbf{P}^{1}$ for $s \neq o$ (see [6]); however, as we shall see, $X_{o} \not X_{s}(s \neq o$ ). In fact, if $\Theta$ denotes the sheaf of germs of holomorphic tangent vector fields along the fibres, $\Theta_{s}$ the sheaf of germs of holomorphic tangent vector fields on $X_{s}$, we have:

$$
\operatorname{dim} H^{0}\left(X_{0}, \Theta_{0}\right)=7, \quad \operatorname{dim} H^{1}\left(X_{0}, \Theta_{0}\right)=1, \quad \operatorname{dim} H^{2}\left(X_{0}, \Theta_{0}\right)=0,
$$

while

$$
\operatorname{dim} H^{0}\left(X_{s}, \Theta_{s}\right)=6, \quad \operatorname{dim} H^{1}\left(X_{s}, \Theta_{s}\right)=0, \quad \operatorname{dim} H^{2}\left(X_{s}, \Theta_{s}\right)=0,
$$

if $s \neq o$. These results are obtained by a direct computation using Čech theory.

Since $H^{2}\left(X_{0}, \Theta_{0}\right)=0$, Corollary to Proposition 2.1 shows that no class in $H^{1}\left(X_{0}, \Theta_{0}\right)$ is obstructed. We see, therefore, that the criterion of Corollary 1 to Theorem 4.1 is not necessary for no obstructions.

We must expect, on the other hand, to find obstructed classes in $H^{0}\left(\mathrm{X}_{0}, \mathrm{O}_{0}\right)$. for otherwise, Proposition 4.2 and Theorem 4.4 would show that $\operatorname{dim} H^{1}\left(X_{s}, \Theta_{s}\right)$ would be independent of $s$ in a neighborhood of o (which we know is not the case). We will explicitly calculate $H^{0}\left(X_{0}, \Theta_{0}\right)$ and show the obstructed classes.

$X$ may be covered by 4 open sets each isomorphic to $\mathbf{C}^{3}: U_{i}=U_{i} \times \mathbf{C}$. $(i=1, \ldots, 4)$. Here $U_{i} \cong \mathrm{C}^{2}$ with coordinates $\left(z_{1}, \zeta_{i}\right)$. The coordinate changes are given by 


$$
\begin{array}{ll}
z_{1}=1 / z_{2}, & \zeta_{1}=z_{2 \succ_{2}}^{2}+s z_{2}, \\
z_{i}=z_{i+2}, & \zeta_{i}=1 / \zeta_{i+2} \quad(i=1,2) .
\end{array}
$$

$\left\{\mathcal{U}_{i}\right\}$ is a Stein cover, so the $\breve{C}$ ecn groups for $\mathcal{U}$ are the cohomology groups of $X$. Direct computation shows that $H^{0}\left(X_{0}, \Theta_{0}\right)$ consists of $\theta=\left\{\theta_{i}\right\}$ such that

$$
\theta_{1}=\left(a+b z_{1}+c z_{1}^{2}\right)\left(\partial / \partial z_{1}\right)+\left[\left(d-2 c z_{1}\right) \zeta_{1}+\left(e+f z_{1}+g z_{1}^{2}\right) \zeta_{1}^{2}\right]\left(\partial / \partial \zeta_{1}\right),
$$

$a, \cdots, g$ being arbitrary complex numbers. $\theta$ is obstructed unless $b+d=0$. In the unobstructed case, an extension of $\theta$ is given by

$$
\begin{aligned}
\theta_{1}=\left(a(s)+b(s) z_{1}+c(s) z_{1}^{2}\right)\left(\partial / \partial z_{1}\right) & \\
& +\left[\left\{c(s) s+g(s) s^{2}\right\}+\left\{(-b(s)-f(s) s)+(-2 c(s)-2 g(s) s) z_{1}\right\} \zeta_{1}\right. \\
& \left.+\left\{e(s)+f(s) z_{1}+g(s) z_{1}^{2}\right\} \zeta_{1}^{2}\right]\left(\partial / \partial \zeta_{1}\right)
\end{aligned}
$$

where $a(s), b(s), c(s), e(s), f(s), g(s)$ are arbitrary holomorphic functions of $s$ such that $a(0)=a, b(0)=b=-d, c(0)=c, e(0)=e, f(0)=f, g(0)=g$.

\section{REFERENCES}

1. J. Frisch, Points de platitude d'un morphisme d'espaces analytiques complexes, Invent. Math. 4 (1967), 118-138. MR 36 \#5388.

2. R. Godement, Théorie des faisceaux, Hermann, Paris, 1958.

3. H. Grauert, Ein Theorem der analytischen Garbentheorie und die Modulräume komplexer Strukturen, Inst. Hautes Études Sci. Publ. Math. No. 5 (1960). MR 22 \#12544.

4. P. A. Griffiths, The extension problem for compact submanifolds of complex manifolds. I. The case of a trivial normal bundle, Proc. Conf. Complex Analysis (Minneapolis, 1964), Springer,-Verlag, New York, 1965. MR 32 \#8362.

5. A. Grothendieck, Éléments de géométrie algébrique, Publ. Inst. Hautes Études Sci. Publ. Math. 1960-1970.

6. K. Kodaira, On stability of compact submanifolds of complex manifolds, Amer. J. Math. 85 (1963), 79-94. MR 27 \#3002.

7. O. Riemenschneider, Über die Anwendung algebraisher Methoden in der Deformationstheorie komplexer Räume, Math. Ann. 187 (1970), 40-55. MR 71 \#5659.

8. J. J. Wavrik, Obstructions to the existence of a space of moduli, Global Analysis (Papers in Honor of K. Kodaira), Univ. Tokyo Press, (1969), pp. 403-414. MR 40 \#8089.

9. - A theorem of completeness for families of compact analytic spaces, Trans. Amer. Math. Soc. 163 (1972), 147-155.

DEPARTMENT OF MATHEMATICS, UNIVERSITY OF CALIFORNIA AT SAN DIEGO, LA JOLLA, CALIFORNIA 92037 